\title{
Prototipagem e cirurgia guiada em implantodontia: revisão de literatura
}

\section{Prototyping and guided surgery in implantology: literature review}

\author{
Jefferson da Rocha Tenório* \\ Eliziane Sampaio de Souza* \\ Marleny Elizabeth Martinez Gerbi** \\ Belmiro Cavalcanti do Egito Vasconcelos ${ }^{* * *}$
}

\section{Resumo}

Objetivo: o objetivo deste estudo é fazer uma revisão de literatura descrevendo os benefícios, as vantagens e desvantagens de modelos de prototipagem para o planejamento e a redução do tempo em cirurgia. Revisão de literatura: a prototipagem rápida é uma tecnologia que permite a duplicação morfológica de estruturas anatômicas em escala real, obtidas por meio de exames de imagem, resultando nos chamados biomodelos, que permitem a visualização tridimensional das estruturas anatômicas complexas, facilitando o planejamento pré-operatório de implantes. Considerações finais: a prototipagem possui amplo uso em odontologia, mais especificamente no campo da implantodontia e cirurgia bucomaxilofacial. Consiste em uma técnica aplicável, pois permite a realização da cirurgia guiada. Bem como esses protótipos permitem a redução do tempo cirúrgico, do tempo necessário de anestesia e do risco de infecção. Em Implantodontia, a técnica da prototipagem facilita a obtenção dos guias cirúrgicos, tornando-se um diferencial para que se consiga um perfeito planejamento reverso, ou seja, uma boa dinâmica e estética agradáveis. A prototipagem e a cirurgia guiada representam um novo horizonte na implantodontia de forma que os procedimentos cirúrgicos se tornam mais simples, seguros e previsíveis. Com esse tipo de procedimento é possível otimizar o tempo da intervenção cirúrgica e ainda gerar um maior grau de confiança, devido às simulações e informações obtidas pelos biomodelos.

Palavra-chave: Projeto auxiliado por computador. Implantes dentários. Cirurgia bucal.

\section{Introdução}

A prototipagem tem se constituído num recurso de grande importância para o planejamento cirúrgico-protético em situações de alta complexidade, como as encontradas na cirurgia bucomaxilofacial e na implantodontia ${ }^{1}$. Na Odontologia, as tecnologias $\mathrm{CAD} / \mathrm{CAM}$ (computer-aided desing (CAD) / computer-aided manufacturing (CAM)) têm sido ampliadas no campo das próteses desde os anos 1980. Mais especificamente, a aplicação da tecnologia CAD/ CAM na Odontologia Restauradora e Prótese Fixa têm crescido consideravelmente nos últimos anos ${ }^{2}$.

As tecnologias auxiliadas por computadores permitem uma modelagem mais precisa e sistemática e podem, dessa forma, reduzir passos morosos na cadeira odontológica e do trabalho de laboratório, otimizando o tempo de trabalho ${ }^{3}$. A Prototipagem Rápida (PR) é uma tecnologia CAD/CAM que foi desenvolvida, inicialmente, para fabricação de protótipos para fins industriais. Esse método constrói, automaticamente, modelos físicos de arquivos computadorizados em 3D. A PR tem recentemente mostrado aplicações bem sucedidas em vários campos odontológicos, como na fabricação de guias cirúrgicos de implantes, prótese maxilofacial e estrutura para prótese parcial removível ${ }^{4}$. A manufatura de dentaduras completas da forma tradicional foi introduzida cerca de 70 anos atrás, quando era dependente de habilidades proficientes de dentistas e técnicos em Odontologia ${ }^{5}$.

\footnotetext{
Cirurgião-dentista pela Universidade de Pernambuco, Faculdade de Odontologia de Pernambuco, Departamento de Implantodontia, Camaragibe, PE, Brasil.

- Doutora Universidade de Pernambuco, Faculdade de Odontologia de Pernambuco, Departamento de Implantodontia, Camaragibe, PE, Brasil.
}

* Doutor Universidade de Pernambuco, Faculdade de Odontologia de Pernambuco, Departamento de Implantodontia, Camaragibe, PE, Brasil. 
A prototipagem rápida é uma tecnologia que permite a duplicação morfológica de estruturas anatômicas em escala real de 1:1, no sistema CAD (computer-aided design) obtidas por meio de exames como a tomografia computadorizada, a ressonância magnética e a ultra-sonografia, resultando nos chamados biomodelos, que permitem a visualização tridimensional das estruturas anatômicas complexas, facilitando o planejamento pré-operatório ${ }^{6}$.

A prototipagem é uma técnica aplicável, pois permite a realização da cirurgia guiada. Por meio dela é possível o diagnóstico e o tratamento, em que se planeja a intervenção, pela construção de guias cirúrgicas personalizadas. Com a prototipagem ocorre uma diminuição da agressão ao tecido, com isso, reduz-se o tempo cirúrgico e tem-se ainda uma menor contaminação das áreas cirúrgicas, possibilitando, dessa forma, uma cicatrização mais rápida ao paciente ${ }^{6}$.

O presente trabalho tem como objetivo fazer uma revisão de literatura descrevendo os benefícios, as vantagens e as desvantagens de modelos de prototipagem para o planejamento e a redução de tempo em cirurgia.

\section{Revisão de literatura}

\section{Histórico}

A fabricação de protótipos é um marco nas Engenharias, considerado um passo intermediário entre os estudos prévios de desenvolvimento e o produto final, auxiliar na avaliação da viabilidade dos projetos, evitando custos excessivos e possíveis defeitos de fabricação ${ }^{7}$.

A era da osseointegração teve seu início a partir dos trabalhos publicados pelo professor Branemark no início da década de 1980. Todo o planejamento dos casos a serem reabilitados com implantes osseointegráveis tinha como passo inicial a realização e a análise de exames radiográficos convencionais, que influenciavam principalmente na determinação do comprimento dos implantes, considerando a altura óssea disponível. Entretanto, decisões importantes como o posicionamento, a distribuição e as inclinações dos implantes eram tomadas apenas durante o trans-cirúrgico, considerando a análise do tecido ósseo disponível, o que, muitas vezes, levou à ocorrência de insucessos estéticos e funcionais ${ }^{8}$.

$\mathrm{Na}$ área da saúde, tem-se utilizado rotineiramente, nos últimos anos, o planejamento pré-cirúrgico baseado em exames de Tomografia Computadorizada e Ressonância Magnética. Por meio dessas técnicas é possível reproduzir, tridimensionalmente, estruturas, porém exibindo-as em superfícies bidimensionais (ex: tela do computador), esse fato as torna representações da estrutura em 3D e não uma estrutura verdadeiramente tridimensional ${ }^{9}$.

\section{Prototipagem}

Os Biomodelos são Protótipos biomédicos obtidos a partir de imagens tomográficas Computadorizadas Axiais (TCA), que têm como finalidade auxiliar no tratamento das deformidades ${ }^{10}$.

Os protótipos vão permitir a obtenção de medidas das estruturas, simulação e planejamento cirúrgicos, diminuindo dessa forma, o tempo cirúrgico, o tempo de anestesia e o risco de infecção ${ }^{11}$.

Para obtenção de um protótipo, de acordo com Carvalho ${ }^{12}$, (2007), seguem-se as seguintes etapas:

- exame tomográfico computadorizado helicoidal ou volumétrico da região com cortes reformatados em um milímetro. A espessura dos cortes é o que determina a qualidade do modelo;

- gravar as imagens no formato DICOM (Digital Imaging and Communications in Medicine) em uma das diversas mídias existentes;

- enviar a mídia com as imagens para a empresa que produz o protótipo, podendo também ser enviada pela internet via FTP (File Transfer Protocol);

- obtenção do protótipo Biomodelo.

Existem varias técnicas de prototipagem rápida, para obtenção de biomodelos. As mais utilizadas são Estereolitografia (SLA), a Sinterização Seletiva a Laser (SLS) A Impressão Tridimencional (3D printing), a Modelagem por Deposição Fundida (FDM), a PolyJet e a "Thermojet" (MJM). Todas baseiam-se na sinterização, aglutinação, polimerização ou solidificação de materiais específicos ${ }^{13}$.

$\mathrm{Na}$ Odontologia, a prototipagem tornou-se útil na implantodontia, na cirurgia bucomaxilofacial, sendo menos utilizada no planejamento ortodôntico, para analisar o posicionamento real dos dentes ${ }^{14}$.

$\mathrm{O}$ advento da prototipagem rápida como ferramenta de modelagem anatômica e os avanços da tomografia computadorizada contribuíram para um aumento na precisão das cirurgias de implante ${ }^{15}$.

\section{Cirurgia guiada}

Em Odontologia, mais especificamente na implantodontia e cirurgia bucomaxilofacial, cada vez mais os pacientes apresentam atrofia óssea, logo necessitam de enxertos ósseos. Diante disso, se torna muito difícil prever resultados, o que torna uma cirurgia guiada por protótipos recomendada, pois consegue-se diminuir o tempo cirúrgico, a agressão ao tecido e ainda ter uma menor contaminação das áreas cirúrgicas adjacentes, possibilitando uma cicatrização mais rápida para o paciente ${ }^{16}$.

Em implantodontia, a utilização de um guia cirúrgico permite uma melhor emergência possível na prótese, isso depois de uma perfeita montagem dos dentes em cera e com a verificação da fonéti- 
ca, da oclusão e do corredor bucal. O guia cirúrgico é um diferencial para que se consiga um perfeito planejamento reverso, ou seja, dinâmica e estética agradáveis ${ }^{17}$.

A confecção do guia cirúrgico antes da cirurgia possibilita ao implantodontista durante a implantação respeitar os limites mesio-distal, vestíbulo-lingual e ápico-coronal ${ }^{16,17}$.

\section{Discussão}

A técnica da PR tem sido aplicada em diversas áreas da odontologia, possibilitando aos profissionais incluir essa técnica como parte de seus recursos de diagnóstico em situações clínicas que vão desde a pesquisa e análise de patologias, perpassando por avaliação da anatomia óssea, detecção de fraturas da face, avaliação das articulações temporomandibulares, seios paranasais, abrangendo, ainda o plano de tratamento implantodôntico ${ }^{8}$.

Chaware et al. ${ }^{6}$ (2009) afirmaram que técnica de prototipagem permite a construção de guias cirúrgicos personalizados na evidenciação de discrepâncias maxilomandibulares, ou seja, permite a duplicação morfológica de estruturas anatômicas complexas em escala real de 1:1 com visualização tridimensional, tornando-se totalmente fidedigna. Os resultados publicados neste estudo informaram que os implantes têm $98 \%$ dos resultados positivos, quando há estabilidade no tratamento, evitando riscos e inconveniências maiores aos pacientes e desconfortos.

Rosa et al. ${ }^{18}$ (2004) afirmaram que a pototipagem é uma técnica usada para produzir modelos em imagens digitalizadas, assim como a tomografia computadorizada. Para os autores, a prototipagem na cirurgia segue por dois estágios: o virtual, simulando o trajeto do implante, e o físico, que é a fabricação do modelo de prototipagem que consiste em usar o processo de imagem como ferramenta de constituição de dimensões físicas ao longo da cirurgia. Os autores aduzem que a essa dimensão física da prototipagem constitui um modelo criado no computador e construído passo a passo ao longo de outro modelo prévio. Essa tecnologia aplicada na cirurgia guiada teve resultados positivos.

Thomé et al. ${ }^{19}$ (2009) afirmaram que a técnica da cirurgia guiada apresenta muitas vantagens se comparada às técnicas convencionais. Entre elas podemos citar o conforto para o paciente, a ausência de sintomatologia dolorosa e de edemas pós-cirúrgicos, a redução do tempo da cirurgia e a redução da quantidade de anestésicos e medicamentos em geral. É uma técnica viável e simples, pois apresenta um grau de dificuldade maior quando se deseja obter a prótese previamente à cirurgia, pois todos os detalhes devem ser bastante precisos. O posicionamento de cada implante e a seleção dos pilares com o auxílio do software devem ser bem planejados ${ }^{19}$.
Viana Neto et al. ${ }^{20}$ (2009) relatam um caso de cirurgia guiada virtual sem retalho, na qual foi realizada a reabilitação de uma maxila edêntula. $\mathrm{O}$ paciente de 42 anos tinha como queixas, a estética e a dificuldade mastigatória. $\mathrm{O}$ planejamento inclui, além da instalação de quatro implantes, com auxílio da guia virtual, a reabilitação com prótese total provisória imediata. $\mathrm{O}$ paciente demonstrou, após o procedimento, satisfação estética e funcional. Os autores relatam, além das vantagens já citadas, a facilidade da realização do procedimento e salienta a necessidade da realização de estudos em longo prazo, à cerca da técnica.

Freitas Filho $^{16}$ (2010) analisou por meio de revisão de literatura a importância da PR no planejamento das reabilitações com implantes zigomáticos. As principais vantagens com a utilização dos biomodelos são: aumento da previsibilidade, a redução de $30 \%$ do tempo cirúrgico, a redução do período de anestesia e do risco de infecção e a diminuição do custo total do tratamento.

Kawakami et al. ${ }^{21}$ (2011) afirmaram que outra opção de planejamento que aumenta a precisão da técnica de instalação das fixações zigomáticas é a utilização de guias cirúrgicos confeccionados por meio de prototipagens obtidas após tomografias computadorizadas com reconstruções 3D em volume. Esses guias apresentam grande fidelidade. Além disso, as prototipagens podem ser importantes no planejamento, no treinamento e na simulação cirúrgica das fixações zigomáticas. Por fim, podem-se associar os exames clínicos com os exames de imagem e fazer também o uso de modelos montados em articulador e do enceramento diagnóstico, permitindo uma previsibilidade dos resultados.

Muerer et al. ${ }^{22}$ (2008) demonstraram que a prototipagem nos eventos cirúrgicos inova os conceitos de reabilitação, apontando possíveis soluções em um tempo mínimo. Os protótipos adotados detectam as correções necessárias e reconstroem o objeto da reabilitação com estruturas anatômicas, manipuladas em um programa computadorizado, pela prototipagem rápida. $\mathrm{O}$ processo de prototipagem garante intensa interação com a tecnologia e o processo de imagem. A cirurgia guiada com a prototipagem exploram a informação aos cuidados de radiologistas específicos. Este estudo apresenta as ferramentas necessárias ao processo de cirurgia guiada com a prototipagem e a integração de ambos com os cirurgiões e especialistas em radiologia em prol dos resultados: as imagens, que constituirão a prévia e determinarão a duração do procedimento cirúrgico. A prototipagem, para esses autores, é um processo que reduz o custo da cirurgia e elimina fases de risco para o paciente. A manipulação, a conversão e o trabalho das imagens irão compor informações importantes para a qualidade de tempo e de procedimento dos profissionais. Ainda é afirmado que a visualização direta da reconstrução do objeto requer a simulação de estrutura osteo-anatômica 
como prévia das aplicações de técnicas cirúrgicas. A prototipagem previne estágios em que há riscos de infecção para o paciente. Os procedimentos cirúrgicos obtêm produções úteis para os implantes dentários.

Cada uma dessas novas técnicas necessita de mais investigações e acompanhamentos, principalmente no que se refere a casos de intensa atrofia óssea. Esses são os aspectos mais importantes dessa tecnologia que podem contribuir para o estabelecimento do padrão de alta qualidade da implantodontia ${ }^{23}$.

\section{Considerações finais}

A prototipagem e a cirurgia guiada representam um novo horizonte na implantodontia e na cirurgia buço-maxilo-facial, em que os procedimentos cirúrgicos se tornam mais simples, seguros e previsíveis. Com esse tipo de procedimento é possível otimizar o tempo do paciente sob intervenção cirúrgica e ainda gerar um maior grau de confiança devido às simulações e informações obtidas pelos biomodelos.

\section{Abstract}

Objective: The objective of this study is to perform a literature review describing the benefits, advantages, and disadvantages of prototyping models for the planning and reduction of surgical time. Literature review: Rapid Prototyping is a technology that allows the morphological duplication of anatomical structures in real scale, obtained by imaging analysis and resulting in the so-called biomodels, which allow tridimensional visualization of complex anatomical structures, facilitating the pre-operative planning of implants. Final Considerations: Prototyping is widely used in Dentistry, more specifically in implantology and oral maxillofacial surgery. It is an applicable technique that allows the performance of guided surgery. These prototypes also allow the reduction of the surgical time, time of anesthesia required, and the risk of infection. In implantology, the prototyping technique facilities the attainment of surgical guides, which is a differential for obtaining a perfect reverse planning, that is, good dynamics and pleasant esthetics. Prototyping and guided surgery represent a new horizon in implantology, where surgical procedures become easier, safer, and more predictable. This procedure enables optimizing the time of surgical intervention, and creating a higher degree of confidence due to the simulations and information obtained by the biomodels.

Keywords: Computer-aided design. Dental implants. Oral surgery.

\section{Referências}

1. Inokoshi M, Kanazawa M, Minakuchi S. Evaluation of a complete denture Trial method applying rapid prototyping. Dent Mat J 2012;31(1):40-6.

2. Miyazaki T, Hotta Y, Kunii J, Kuriyama S, Tamaki Y. A review of dental CAD/CAM: current status and future perspectives from 20 years of experience. Dent Mater 2009;(28):44-56.

3. Kanazawa M, Inokoshi M, Minakuchi S, Ohbayashi N. Trial of a $\mathrm{CAD} / \mathrm{CAM}$ system for fabricating complete dentures. Dent Mater J 2011;(30):93-6.

4. Lo J, Chen Y. Three-Dimensional computed tomography imagin in craniofacial surgery: morphological study and clinical applications. Chang Gung Med J 2003;26(1):1-11.

5. Stoetzer M, Rana M, See C, Eckard AM, Gellrich NC. Reconstrution of defects of maxillary sinus wall after removal of a huge odontogênica lesion using prebend 3D titanium-mesh and CAD/CAM technique. Head \& Face Med 2011;7(21):1-3.

6. Chaware SM, Bagaria V, Kuthe A. Aplication of the rapid prototyping techine to design a customized temporomandibular joint used to treat temporomandibular ankylosis. Implant News 2005;2(2):153-60.

7. Russett S. Major P, Toogood R, Boulanger P. An Experimental method fot stereolithic mandible fabrication and image preparation. The Open Biomed Engi J 2007;(1):1-8.

8. Menezes PDF, Sarmento V, Lamberti P. Aplicação da prototipagem rápida em implantodontia. Innovations Imp J Biomaterials and Esthetics. 2008;3(6):1-12.

9. Jackson I, Xiao H, Ashtiani M, Berben L. Stereolithography model in presurgical planning of craniofacial surgery. 2009 [acesso em: 27 out. 2012]. P. 9-14. Disponível em URL: http://utwired.engr.utexas.edu/lff/symposium/proceedingsArchive/pubs/Manuscripts/1996/1996-02-Jackson.pdf.

10. Benazzi S, Fiorenza L, Kozakowski, S, Kullmer O. Comparing $3 \mathrm{D}$ virtual methods for hemimandibular body reconstruction. J Oral Maxilofac Surg. 2000;58(3):349-51.

11. Meurer E, Oliveira MG, Meurer MI, Silva JVL, Bárbara AS, Heitz C. Os biomodelos de prototipagem rápida em cirurgia e traumatologia bucomaxilofacial. Academia Tiradentes de Odontologia 2007;5:349-73.

12. Carvalho EM. Prototipagem na Odontologia: obtenção e uso. In: Congresso Internacional de Técnicos em Prótese Dentária, 10. 11 a 13 de outubro de 2007. São Paulo; 2007.

13. Sanghera B, Naique S, Papaharilaou Y. Preliminary suty of rapid prototype medical models. Rapid Prototyping J 2001;7(5):275-84.

14. Balem FP. A utilização de Protipagem Rápida em Odontologia [Tese de Doutorado]. Porto Alegre: Universidade Federal do Rio Grande do Sul; 2010.

15. Mcallister MLM. Application of stereolithography to subperiosteal implant manufacture. J Oral Implantol 1998;24(2):89-92.

16. Freitas Filho AR. Protipagem em Implantodontia e Cirurgia Guiada [Tese de Doutorado]. Rio de Janeiro: CIODONTO/ FAISA; 2010.

17. Thomé G, Hermann C, Thomé JGP, Sartori IAM, Melo ACM. O uso da cirurgia guiada na reabilitação unitária em região estética. J ILAPEO 2009;3(3):1-5.

18. Rosa ELS, Oleskovicz CF, Aragão BN. Rapid prototyping in maxillofacial surgery and traumatology: case report. Braz Dent J 2004;15(3):243-7.

19. Thomé G, Borges AFS, Bernades SR, Golin AL, Buche A. Soluções virtuais para problemas reais. Jornal do ILAPEO 2009;3(3):6-13. 
20. Viana AV, Neves PJC, Madruga FATTA, Rocha RS, Carvalho RWF. Cirurgia guiada virtual para reabilitação oral: revisão de literatura e relato de caso. Rev Cir Traumatol Buco-maxilo-facial 2009;9(2):45-52.

21. Kawakami PY, Ferrari RB, Silva Neto UT, Almeida TE, Nascimento KG. Implantes zigomáticos: revisão de literatura com estudo do índice de sucesso através de meta-análise. Rev Odontol Univ da Cidade de São Paulo 2011;23(1):51-8.

22. Muerer MI, Muerer ES, Jorge VB, Ailton N, Luiz Felipe O, Marilia SD. Aquisition and manipulation of computed tomography images of the maxillofacial region for biomedical prototyping. Radiol Bras 2008;41(1):49-54.

23. Pozzi A, De Vico G, Sannino G, Spinelli D, Ottria L, Barlattani A.Flapless trascrestal Maxillary sinus floor elevation: computer guided implant surgery combined with expanding-condensing osteotomes protocol. Oral \& Implantology 2011;4(1):4-9.

Endereço para correspondência:

Marleny Elizabeth Martinez Gerbi

Av. General Newton Cavalcanti, 1650, Tabatinga

54.753-220 Camaragibe/PE

Telefone/fax: (81) 3184-7674

E-mail: marleny_gerbi@msn.com.br

Recebido: 24/10/2013. Aceito: 28/04/2015. 